\title{
MoMyb1 is required for asexual development and tissue-specific infection in the rice blast fungus Magnaporthe oryzae
}

\author{
Yanhan Dong, Qian Zhao, Xinyu Liu, Xiaofang Zhang, Zhongqiang Qi, Haifeng Zhang*, Xiaobo Zheng \\ and Zhengguang Zhang
}

\begin{abstract}
Background: The Myb super-family of proteins contain a group of functionally diverse transcriptional activators found in plant, animal and fungus. Myb proteins are involved in cell proliferation, differentiation and apoptosis, and have crucial roles in telomeres. The purpose of this study was to characterize the biological function of Myb1 protein in the rice blast fungus Magnaporthe oryzae.

Results: We identified the Saccharomyces cerevisiae BAS1 homolog MYB1 in M. oryzae, named MoMyb1. MoMyb1 encodes a protein of 322 amino acids and has two SANT domains and is well conserved in various organisms. Targeted gene deletion of MOMYB1 resulted in a significant reduction in vegetative growth and showed defects in conidiation and conidiophore development. Quantitative RT-PCR analysis revealed that the transcription levels of several conidiophore-related genes were apparently decreased in the $\Delta$ Momyb1 mutant. Inoculation with mycelia mats displayed that the virulence of the $\Delta$ Momyb1 mutant was not changed on rice leaves but was non-pathogenic on rice roots in comparison to the wild type Guy 11. In addition, $\triangle M$ Momyb1 mutants showed increased resistance to osmotic stresses but more sensitive to cell wall stressor calcofluor white (CFW). Further analysis revealed that MoMyb1 has an important role in the cell wall biosynthesis pathway.
\end{abstract}

Conclusion: This study provides the evidence that MoMyb1 is a key regulator involved in conidiogenesis, stress response, cell wall integrity and pathogenesis on rice roots in the filamentous phytopathogen M. oryzae.

Keywords: Magnaporthe oryzae, Conidiogenesis, Stress response, Cell wall integrity, Pathogenesis

\section{Background}

Rice blast caused by the heterothallic ascomycete Magnaporthe oryzae, is the most destructive disease of cultivated rice worldwide and can lead to severe losses of annual rice yield [1,2]. Under normal conditions, the fungus uses a highly specialized infection structure appressorium generated from a conidium for plant penetration [3,4]. After successful penetration, the invasive hyphae grow rapidly in the host cells and caused blast lesions. In 5 to 7 days, the pathogen produces numerous conidia from the lesions and initiates a new infection cycle.

\footnotetext{
* Correspondence: hfzhang@njau.edu.cn

Department of Plant Pathology, College of Plant Protection, Nanjing Agricultural University, and Key Laboratory of Integrated Management of Crop Diseases and Pests, Ministry of Education, Nanjing 210095, China
}

Regulation of gene expression at the level of transcription controls many crucial biological processes. A number of different factors, including transcription factors, are essential for the process of transcription. Transcription factors can recognize DNA in a sequence-specific manner and modulate the frequency of initiation of transcription upon binding to specific sites in the promoter of target genes. The transcription factors can be activators, repressors, or both usually display a modular structure named the DNA-binding domain [5]. In M. oryzae, numerous transcription factors were identified and characterized to be important for proper regulation of infection related morphogenesis [6,7]. In our previous study, many transcription factors, including MoCrz1, MoAp1, MoAtf1, MoHac1, MoBzip10, MoSwi6 and MoMsn2 were reported to be involved in hyphal growth, asexual 
development, stress response, infectious growth and virulence by controlling the expression levels of a series of target genes [8-13]. In plants, Myb protein family comprises a large members of transcription factors [14]. The first identified MYB gene was the 'oncogene' v-Myb derived from the avian myeloblastosis virus [15]. Following $\mathrm{v}-\mathrm{Myb}$, a large and growing family of myb-related genes were discovered in a wide variety of eukaryotes including animals, plants, fungi and slime molds [16-18]. The Myb-related proteins contain a DNA-binding domain and generally function in the regulation of cell growth and differentiation, often by co-regulating gene expression along with DNA-binding proteins of other classes $[19,20]$.

Myb proteins play important roles in controlling phenylpropanoid metabolism, cell shape, and hormonal responses during seed development and germination, and cellular proliferation in plants [21]. Additionally, two Myb proteins from fungi, Cdc5 and flbD were also reported to control cell shape [22,23]. In Schizosaccharomyces pombe, the Cdc5p is essential for G2/M progression and Cdc5 family members participate in a novel pathway to regulate G2/M progression [22,24]. In Aspergillus nidulans, flbD encodes a Myb-like DNA-binding protein and is required for early conidiophore development by activating a cascade of transcription factors for conidiophore production [22,23]. Here, we investigate the role of MoMYB1 in growth and infection-related morphogenesis in M. oryzae. Deletion of MoMYB1 resulted in a failure to develop conidiophores and conidia, and more tolerance to osmotic stressors. Furthermore, MoMyb1 plays a crucial role in cell wall integrity and tissue-specific infection of $M$. oryzae.

\section{Methods}

\section{Fungal strains, cultures and transformation}

The wild type strain Guy11, the mutants and the complemented transformants were cultured on complete medium (CM: $10 \mathrm{~g}$ D-glucose, $2 \mathrm{~g}$ peptone, $1 \mathrm{~g}$ yeast extract, $1 \mathrm{~g}$ casamino acids, $50 \mathrm{ml} 20 \times$ nitrate salts, $1 \mathrm{ml}$ trace elements, $1 \mathrm{ml}$ vitamin solution, $15 \mathrm{~g}$ agar, add distilled water to $1 \mathrm{~L})$ [25], straw decoction and corn powder medium (SDC: $100 \mathrm{~g}$ straw, $40 \mathrm{~g}$ corn powder, $15 \mathrm{~g}$ agar in $1 \mathrm{~L}$ distilled water), V8 juice agar medium

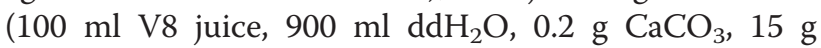
agar) and oatmeal agar medium [26] at $28^{\circ} \mathrm{C}$. Protoplast transformation was performed using hygromycin B $(H P H)$ and bleomycin as selective marker for gene deletion and complementation assays as described [25]. Conidiation assays were performed as described [8].

\section{Deletion and complementation of MoMYB1 in M. oryzae} The MoMYB1 gene deletion mutants were generated using the standard one step gene replacement strategy as described [27]. The primer pairs FL4982/FL4983 and FL4984/FL4985 (Additional file 1: Table S1) were used to amplify the upstream and downstream flanking sequence, respectively. The hygromycin resistance gene cassette was prepared by primer pairs FL1111/FL1112 using pfu Taq DNA polymerase (TaKaRa) (Additional file 1: Table S1). The hygromycin resistant transformants were screened by genomic PCR, and further confirmed by RT-PCR and southern blot analysis. For complementation, the fragment containing the native promoter region and the entire open reading frame (ORF) of MoMYB1 were amplified by primer FL4841/FL4842 (Additional file 1: Table S1) and inserted into the pYF11 vector with a bleomycin resistance gene [28], and then transformed into the $\Delta$ Momyb1 mutant to obtain the complemented transformants.

\section{Pathogenicity assays}

The two-week-old seedlings of susceptible rice cultivar $\mathrm{CO}-39$ were used to perform the detached leaf infection assays. Mycelial plugs of the wild type Guy11, $\Delta$ Momyb1 mutants and the complemented transformant were inoculated on the intact leaves and kept in a moist chamber at $28^{\circ} \mathrm{C}$ for $24 \mathrm{~h}$ in darkness, followed by a $12 / 12$ hour light/dark cycle. Photographs were taken at 7 days after inoculation. Root infection assays were performed as described [29]. Lesion formation was examined at 9 days post-inoculation. The experiments were repeated three times. For infectious hyphal growth on rice roots, mycelia mats of Guy11 and $\triangle M o m y b 1$ expressing a GFP protein were cultured in liquid $\mathrm{CM}$ medium at $28^{\circ} \mathrm{C}$ for 2 days, then harvested and inoculated on the roots. After $48 \mathrm{~h}$ incubation under humid conditions at $28^{\circ} \mathrm{C}$, the roots were observed under a fluorescence microscope.

\section{Osmoregulation and CFW assays}

Osmoregulation and CFW assays were performed as described [30]. Briefly, strain blocks were placed onto the freshly prepared $\mathrm{CM}$ agar plates with $\mathrm{NaCl}(0.7 \mathrm{M}), \mathrm{KCl}$ $(0.6 \mathrm{M})$, and sorbitol $(1 \mathrm{M})$, respectively, and cultured in the dark at $28^{\circ} \mathrm{C}$ for 7 days. For $\mathrm{CM}$ medium containing cell wall perturbing agent Calcofluor White (CFW), the final concentrations were 200, 400, and $600 \mu \mathrm{g} / \mathrm{ml}$ of CFW, respectively. The sensitivity was evaluated by measuring the growth rate, and the experiments were repeated three times with three replicates each time.

\section{Cellular chitin content assay}

Chitin (N-acetylglucosamine, GlcNAc) content was determined as described [31,32]. Mycelia were freeze-dried first. For each sample, $5 \mathrm{mg}$ of dried biomass was resuspended in $1 \mathrm{ml} \mathrm{6 \%} \mathrm{KOH}$ and heated at $80^{\circ} \mathrm{C}$ for $90 \mathrm{~min}$. Samples were centrifuged and pellets washed with PBS and resuspension. The pellets were finally resuspended 
in $0.5 \mathrm{ml}$ of Mcllvaine's buffer with Streptomyces plicatus chitinase (Sigma, USA) and incubated for 16 hours at $37^{\circ} \mathrm{C}$ with gentle mixing. $100 \mathrm{ml}$ sample was then combined with $100 \mathrm{ml}$ of $0.27 \mathrm{M}$ Mosadiumborate, heated for $10 \mathrm{~min}$ at $100^{\circ} \mathrm{C}$, and $1 \mathrm{ml}$ of freshly diluted (1:10) of Ehrlich's reagent was added. After incubating at $37^{\circ} \mathrm{C}$ for $20 \mathrm{~min}, 1 \mathrm{ml}$ of the sample was transferred to a $2.5 \mathrm{ml}$ plastic cuvette (Greiner) and the absorbance at $585 \mathrm{~nm}$ was recorded. Standard curves were prepared with GlcNAc (Sigma, USA). The experiment was repeated three times.

\section{Protoplast release assay}

The wild type Guy11 and the mutant strains were cultured in liquid CM media for 2 days and the mycelia were collected by centrifugation for $10 \mathrm{~min}$ at $5,000 \mathrm{rpm}$. The following lysis and protoplast release steps were performed as described previously [33,34]. The mycelia were washed twice and resuspended using 20\% sucrose. Lysing enzyme from Trichoderma harzianum (Sigma-Aldrich, USA) was added to the suspension, with lysis stopped after 30, 60, and 90 min. Protoplast release were counted with a hemacytometer, and cell wall degradation was examined with the light microscope. The experiment was repeated three times.

\section{Nucleic acid manipulation}

DNA extraction and DNA gel blot hybridization were performed using standard procedures as described [35]. Probe labeling, hybridization and detection were preformed with the DIG High Prime DNA Labeling and Detection Starter Kit (Roche Applied Science, Penzberg, Germany).

\section{Quantitative RT-PCR assay}

Two-week-old rice seedlings were inoculated with a spore suspension of rice blast at $1 \times 10^{5}$ spores $/ \mathrm{ml}$. The inoculated plants were placed in a chamber in the dark for $24 \mathrm{~h}$ at $25^{\circ} \mathrm{C}$, and leaf tissues were collected at $8 \mathrm{~h}$ and $48 \mathrm{~h}$ after inoculation. Mycelia were grown in liquid complete medium for $48 \mathrm{~h}$ at $28^{\circ} \mathrm{C}, 150 \mathrm{rpm}$ and harvested. Total RNA was extracted using the Invitrogen kit as described previously [12].

For quantitative RT-PCR (qRT-PCR), $5 \mathrm{mg}$ of total RNA were reverse transcribed into first-strand cDNA using the oligo (dT) primer and M-MLV Reverse Transcriptase (Invitrogen). The qRT-PCR reactions were performed following previously established procedures [12]. To compare the relative abundance of target gene transcripts, the average threshold cycle $(\mathrm{Ct})$ was normalized to that of ACTIN gene (MGG_03982) as described [36]. $P<0.01$ is used in the statistical test. The primer pairs used in this section are listed in Additional file 1: Table S1.

\section{Bioinformatics}

The full sequence of MoMYB1 was downloaded from the $M$. oryzae online database (http://www.broadinstitute. org/annotation/genome/magnaporthe_grisea/MultiHome. html) [37]. Myb1 sequences from different organisms were obtained from GeneBank (http://www.ncbi.nlm.nih.gov/ BLAST), using the BLAST algorithm [38]. Sequence alignments were performed using the Clustal_W 1.83 program [39].

\section{Results}

\section{Identification of $M$. oryzae MYB1}

The Myb family of proteins is a group of functionally diverse transcriptional activators that is characterized by a conserved DNA-binding domain of approximately 50 amino acids [14]. Here, we identified an S. cerevisiae $B A S 1$ homolog MYB1 from the $M$. oryzae genome by a BLAST_P search. MoMYB1 (MGG_06898.6) encoding a protein of 322 amino acids possesses two SANT (a putative DNA binding domain in the SWI-SNF and ADA complexes, the transcriptional co-repressor $\mathrm{N}-\mathrm{CoR}$ and TFIIIB; InterPro: IPR001005) domains that are interrupted by one intron [40]. Alignment analysis showed that MoMyb1 shares similarities to the homologs from S. cerevisiae, A. nidulans, Schizosaccharomyces pombe, Homo sapiens, Arabidopsis thaliana and Zea Mays [22,23,41-45]. The amino acid sequence identity being $16 \%, 29 \%, 15 \%, 22 \%, 14 \%$, and $21 \%$, respectively. The alignment of repeat DNA-binding domains was shown in Additional file 2: Figure S1. Southern blot analysis revealed MoMYB1 only had a unique copy in $M$. oryzae genome (Additional file 3: Figure S2B)

\section{MoMyb1 is highly expressed during conidia and plant colonization}

To gain insight into the functions of MoMyb1, we first examined the gene expression profile at hyphal, conidial and infectious stages of $M$. oryzae by qRT-PCR. In comparison to the hyphal stage $(1.0 \pm 0.1)$, the expression of MoMYB1 was significantly increased in conidial and infection stages. The abundance of MoMYB1 was increased by 55 -fold $(55.2 \pm 2.6)$ in conidial stage and increased by 40 -fold (40.4 $\pm 8.9,8$ hours post inoculation: hpi) and 71-fold $(71.0 \pm 23.5,48 \mathrm{hpi})$ in the infectious stage (Figure 1).

\section{Targeted deletion of MoMYB1 in M. oryzae}

To evaluate the role of MoMYB1 in growth and development of $M$. oryzae, deletion mutant were generated by replacing the MoMYB1 gene with the hygromycin phosphotransferase resistance cassette (Additional file 3: Figure S2A). Hygromycin resistant transformants were first screened by genomic PCR and further confirmed by southern blot analysis and RT-PCR (Additional file 3: Figure S2B and S2C). Since all successful gene deletion 


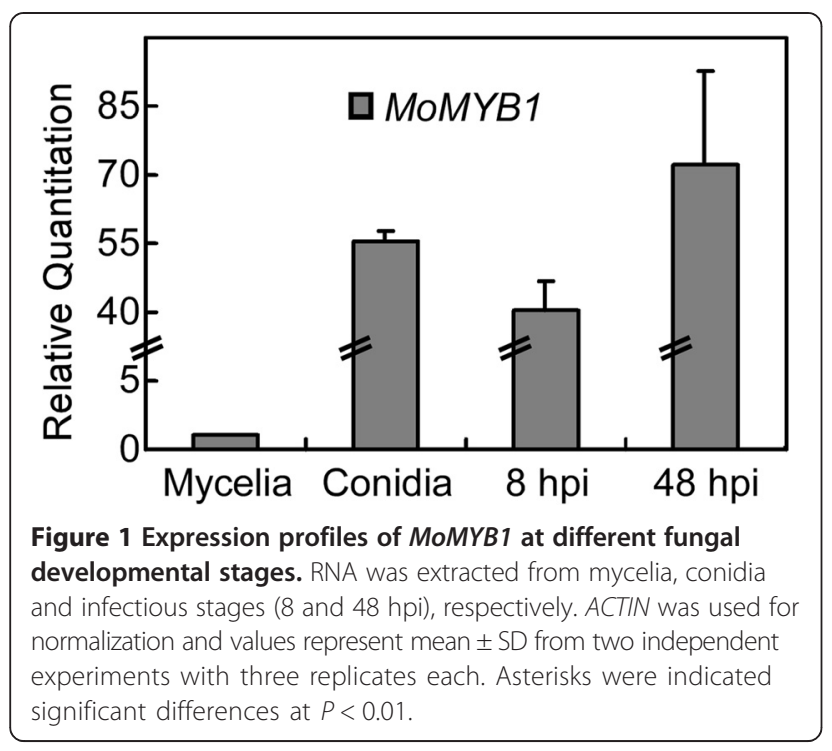

mutants yield the same phenotypes, only one mutant was selected to analyze the biological phenotypes in this study. For complementation, a $2.8 \mathrm{~kb}$ fragment containing the native promoter and ORF of MoMYB1 gene was cloned into pYF11 [28] and reintroduced into the $\Delta M o m y b 1$ mutant. The resulting transformants were confirmed by RT-PCR to obtain the complemented strain $\triangle M o m y b 1 / M o M Y B 1$ (Additional file 3: Figure S2C).

MoMYB1 is involved in vegetative growth and is essential for conidiogenesis

To determine whether MoMYB1 was involved in growth and conidiation, the wild type Guy11, $\triangle M o M y b 1 \mathrm{mu}-$ tants and the complemented transformant $\triangle M o M y b 1 /$ MoMYB1 were inoculated on CM, SDC and V8 agar plates. Compared to Guy11 and $\triangle M o M y b 1 / M o M Y B 1$, the $\Delta M o M y b 1$ mutants showed significant reduced vegetative growth on these three media (Figure 2). The conidiation was also quantified on CM, V8, oatmeal (OM) and SDC media, the result revealed that the production of conidia in the $\Delta M o m y b 1$ mutants was completely abolished on these four media (Figure 3A). To find out the potential reasons of the conidiation defect, we further stained the aerial hyphe with lactophenol cotton blue as described [46], and no conidiophores was observed in the $\Delta M o M y b 1$ mutants, while normal gray conidiophores were formed in the wild type Guy11 (Figure 3B).

\section{MoMYB1 modulates the transcription of several conidiogenesis-related genes}

Because MoMyb1 is a putative Myb transcription factor, which is required for an early stage of conidiation, we speculate that MoMyb1 acts as a transcription factor that regulates the expression of other conidiation-related genes. To test this hypothesis, the expression of several conidiation-related genes or their orthologs was analyzed [11]. The results revealed that MoMSN2, a homologue of ScMSN2 of S. cerevisiae, and MoFLBC, homologous to $F L B C$ of $A$. nidulans, and glutamine synthetase (MoGLUS), homologous to FLUG of A. nidulans, and MoSTUA, homologous to STUA of A. nidulans, and MoCON8, homologous to CON8 of $N$. crassa, was significantly downregulated in the $\triangle M o M y b 1$ mutant (Figure 4).

\section{MoMYB1 plays an essential role in virulence on rice roots but not on leaves}

Since $\Delta$ Momyb1 mutant have a conidiation defect, the mycelial plugs of the wild type Guy11, $\Delta M o m y b 1 \mathrm{mu}$ tant and the complemented transformant were inoculated on the detached rice leaves to test the pathogenic abilities. After 7 days incubation, the $\Delta M o m y b 1$ mutants caused large and extended lesions similar to that of the wild type Guy11 and the complemented transformant (Figure 5A). We further examined the pathogenicity of the $\Delta$ Momyb1 mutant on rice roots. Unlike to the results on rice leaves, the $\Delta M o m y b 1$ mutant caused no virulence on roots 9 days after inoculation. In contrast, the wild type Guy11 and the complemented transformant caused typical rice blast lesions under the same
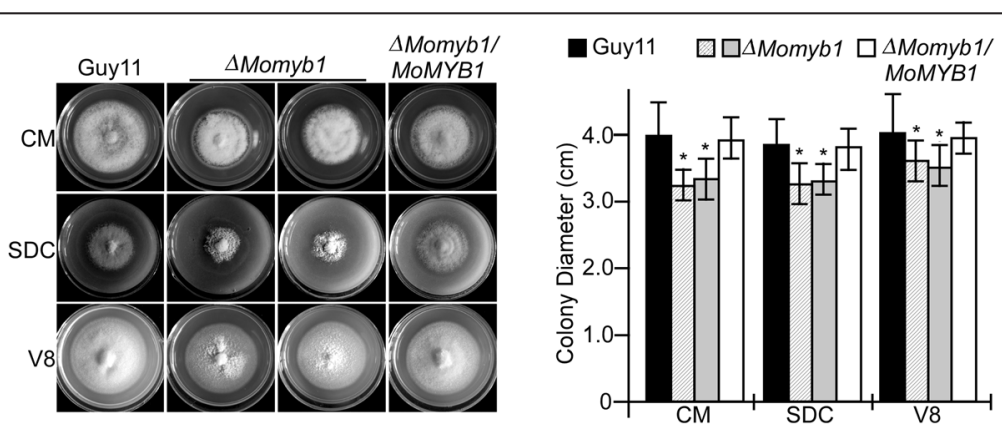

Figure 2 Colony morphology and vegetative growth of Guy11, $\triangle M o m y b 1$ and the complemented transformant $(\triangle M o m y b 1 / M o M Y B 1)$ on complete media (CM), straw decoction and corn (SDC) and 10\% V8 juice agar media (V8). Photographs were taken after 7-day incubation in the dark at $28^{\circ} \mathrm{C}$. Asterisks indicated significant differences at $P<0.01$. Values represent mean $\pm \mathrm{SD}$ from three replicates each. 

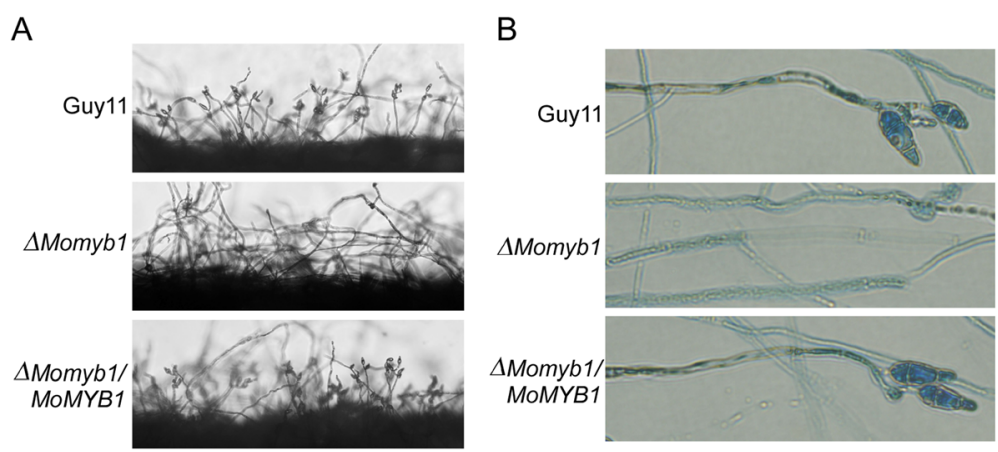

Figure 3 MoMYB1 is required for conidiophore development. (A) Conidia formation was observed under a light microscope 24 hours at room temperature after induction of conidiation on cover slips. The strains were first grown on SDC media for 7 days. (B) Aerial cultures stained with lactophenol cotton blue and observed under light microscope. Hyphae were stained blue, while conidiophores were in gray.

conditions (Figure 5B). These results suggested that MoMyb1 played an important role in tissue-specific infection of $M$. oryzae. To confirm this result, we observed the infectious hyphal growth in the rice root by transforming a green fluorescence protein (GFP) into wild type Guy11 and $\Delta M o m y b 1$ mutant, respectively. The results showed that the wild type could penetrate through the root epidermis and formed branching invasive hyphae at $48 \mathrm{~h}$, while successful penetration and development of invasive hyphae were rarely observed in the $\Delta$ Momyb1 mutant (Figure 5C and D).

Deletion of MoMYB1 results in more insensitive to salt and osmotic stresses

To address the role of MoMYB1 in environmental adaptation, the wild-type Guy11, $\triangle M o m y b 1$ mutant and the

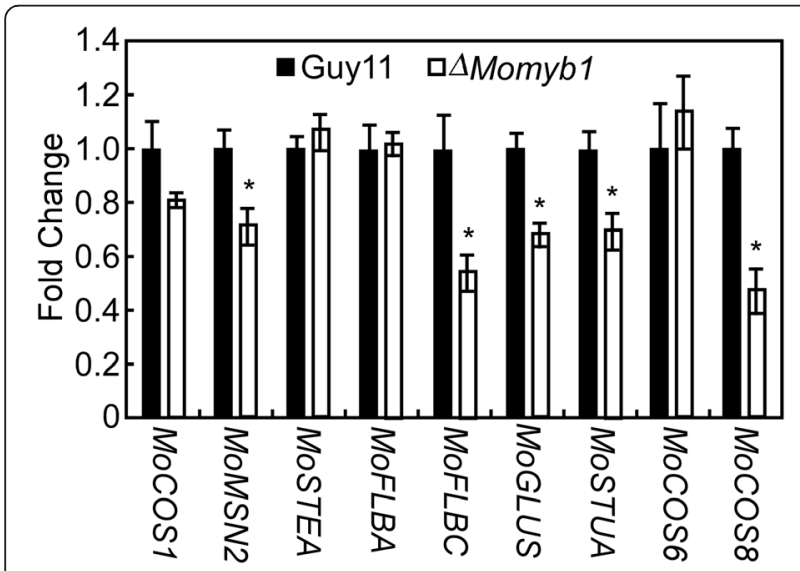

Figure 4 MoMYB1 regulates the transcription of conidiation-related homologous genes. RNA was extracted from mycelia cultured in liquid CM medium at $28^{\circ} \mathrm{C}$ for 2 days. ACTIN was used for normalization, and the values were calculated by $2^{- \text {ddCT }}$ methods with quantitative RT-PCR data. Values represent mean \pm SD from two independent experiments with three replicates each. Asterisks were indicated significant differences at $P<0.01$.

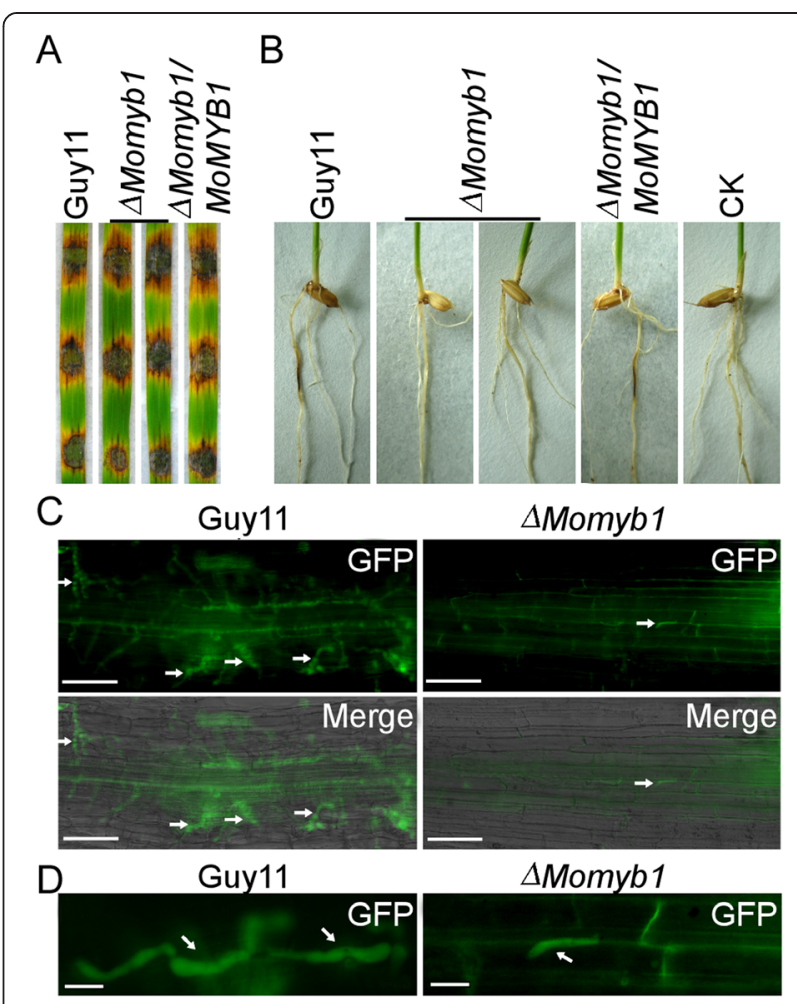

Figure 5 Effect of MoMYB1 deletion on pathogenicity.

(A) Detached rice leaf assay. Intact rice leaves were inoculated by mycelium plugs from wild type Guy 11, $\triangle$ Momyb1 mutant and complemented transformant $\triangle$ Momyb1/MOMYB1. Photographs were taken at 7 days after inoculation. (B) Rice root infection assay. Lesions were examined at 9 days post-inoculation. CK: inoculated with agar plugs without hyphae. (C) Observation of the invasive hyphal growth inside the rice root inoculated with the Guy11 and $\Delta$ Momyb1 strain expression a GFP protein, respectively. White arrows point to invasive hyphae. Bar $=50 \mu \mathrm{m}$. (D) Close view the invasive hyphae in plant cells. White arrows point to invasive hyphae. Bar $=10 \mu \mathrm{m}$. 
complemented transformant were inoculated on the $\mathrm{CM}$ agar plates containing the salt $(0.7 \mathrm{M} \mathrm{NaCl}, 0.6 \mathrm{M} \mathrm{KCl})$ and osmotic (1 M sorbitol) stresses, respectively. Compared with the wild-type and the complemented transformant, the $\Delta M o m y b 1$ mutant showed less sensitivity to $\mathrm{NaCl}, \mathrm{KCl}$ and sorbitol (Figure 6A). The growth rate of the $\Delta M o m y b 1$ mutant was much higher than that of wild type and the complemented transformant (Figure 6B). This result suggested that MoMYB1 has a crucial role in response to salt and osmotic stresses. Since Hog1 pathway was the most important signal pathway to responsible for stress response in M. oryzae [6], we examined the expression of four major components of the pathway, including MoSSK1, MoSSK2, MoPBS2 and MoOSM1 (MoHOG1). The results revealed that, besides MoSSK1, the expression levels of MoSSK2, MoPBS2 and MoOSM1 were significantly decreased in the $\Delta M o m y b 1$ mutant compared to the wild type Guy11 (Figure 6C).

\section{MoMyb1 is involved in cell wall integrity}

To determine whether MoMYB1 has a role in maintenance cell wall integrity, we test mycelial growth on CFW which inhibit fungal cell wall assembly by binding chitin. The results showed that the growth rate of the $\triangle M o m y b 1$ mutant on CFW media was significantly decreased, which reduced to $66.7 \%, 70.8 \%$ and $85.4 \%$ of the wild type Guy11 under 200, 400, and $600 \mu \mathrm{g} / \mathrm{ml}$ CFW, respectively, while the complemented transformant $\triangle M o m y b 1 / M o M Y B 1$ can fully restore the defects
(Figure 7A and B). Chitin is a major component of fungal cell wall and is synthesized by chitin synthases and $M$. oryzae contains seven chitin synthases [47]. Therefore, we examined the transcription levels of these chitin synthase genes by qRT-PCR analysis. The results revealed that the expression of all chitin synthase genes was significantly decreased in the $\Delta M o m y b 1$ mutant (Figure $7 \mathrm{C}$ ). We also examined the chitin content of the mutant and found the chitin content was remarkably reduced in the $\triangle M o m y b 1$ mutant compared to the wild type (Figure 7D), indicating MoMYB1 has a role in cell wall assembly. To further confirm this conclusion, the mycelia of wild type Guy11 and the $\Delta M o m y b 1$ mutant were treated with cell wall degrading enzyme. The results showed that the $\Delta M o m y b 1$ mutant was more sensitive to the enzyme treatment and released more protoplast after incubation for 60 and $90 \mathrm{~min}$ compared to the wild type Guy11 (Figure 8A). When observed at $60 \mathrm{~min}$, the $\Delta$ Momyb1 mutant displayed a greater number of protoplasts and no mycelia fragments were observed. In contrast, the wild type showed much less protoplasts and many mycelial fragments were found under the same condition (Figure 8B).

\section{Discussion}

In the present study, we characterized a Myb family protein, MoMyb1 in M. oryzae and primarily focused on its external phenotypes associated with pathogenesis. Genetargeted replacement revealed that the loss of MoMYB1 led to a plethora of developmental defects in vegetative
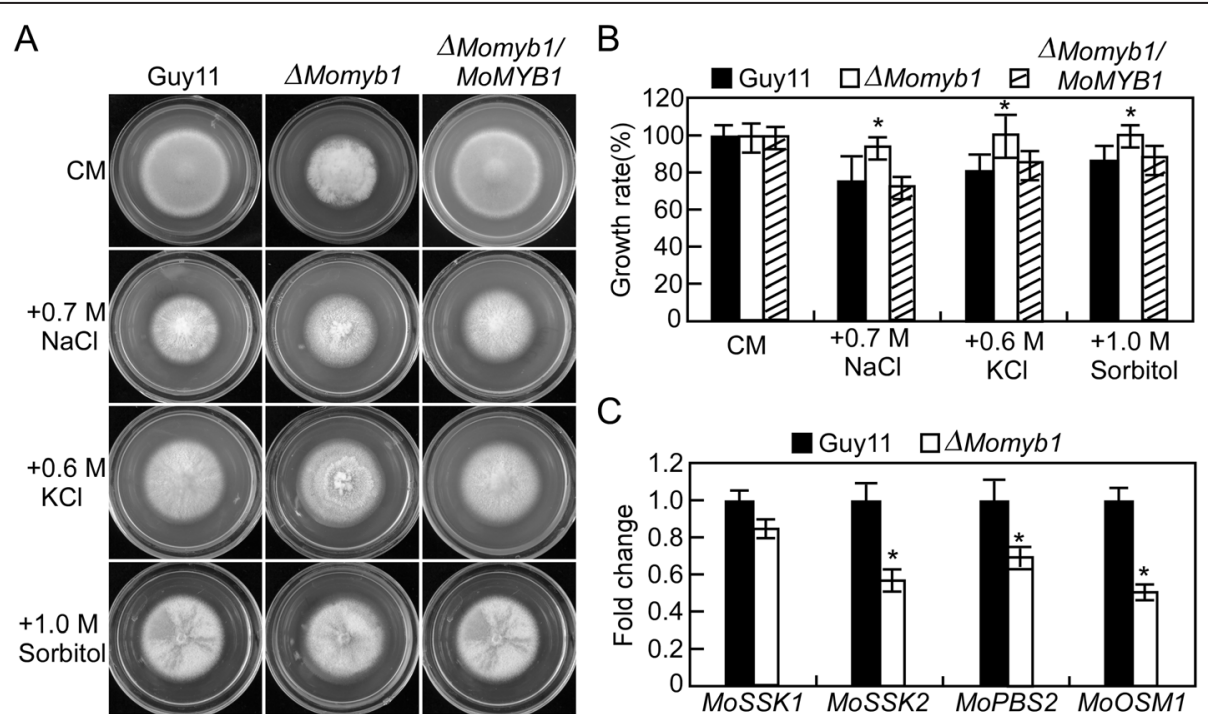

Figure $6 \Delta$ Momyb1 mutants are more insensitive to osmotic stresses. (A) Wild type Guy11, $\Delta$ Momyb1 mutants and complemented transformant were incubated on $\mathrm{CM}$ plates containing various concentrations of $\mathrm{NaCl}, \mathrm{KCl}$ or sorbitol at $28^{\circ} \mathrm{C}$ for 7 days. (B) The growth rate was determined 7 days after incubation at $28^{\circ} \mathrm{C}$ by plotting the percentage of colonies in the presence of various concentrations of $\mathrm{NaCl}, \mathrm{KCl}$ or sorbitol against regular CM. (C) qRT-PCR analysis the transcription of four components of the Hog1 pathway in M. oryzae. Asterisks were indicated significant differences at $P<0.01$. 

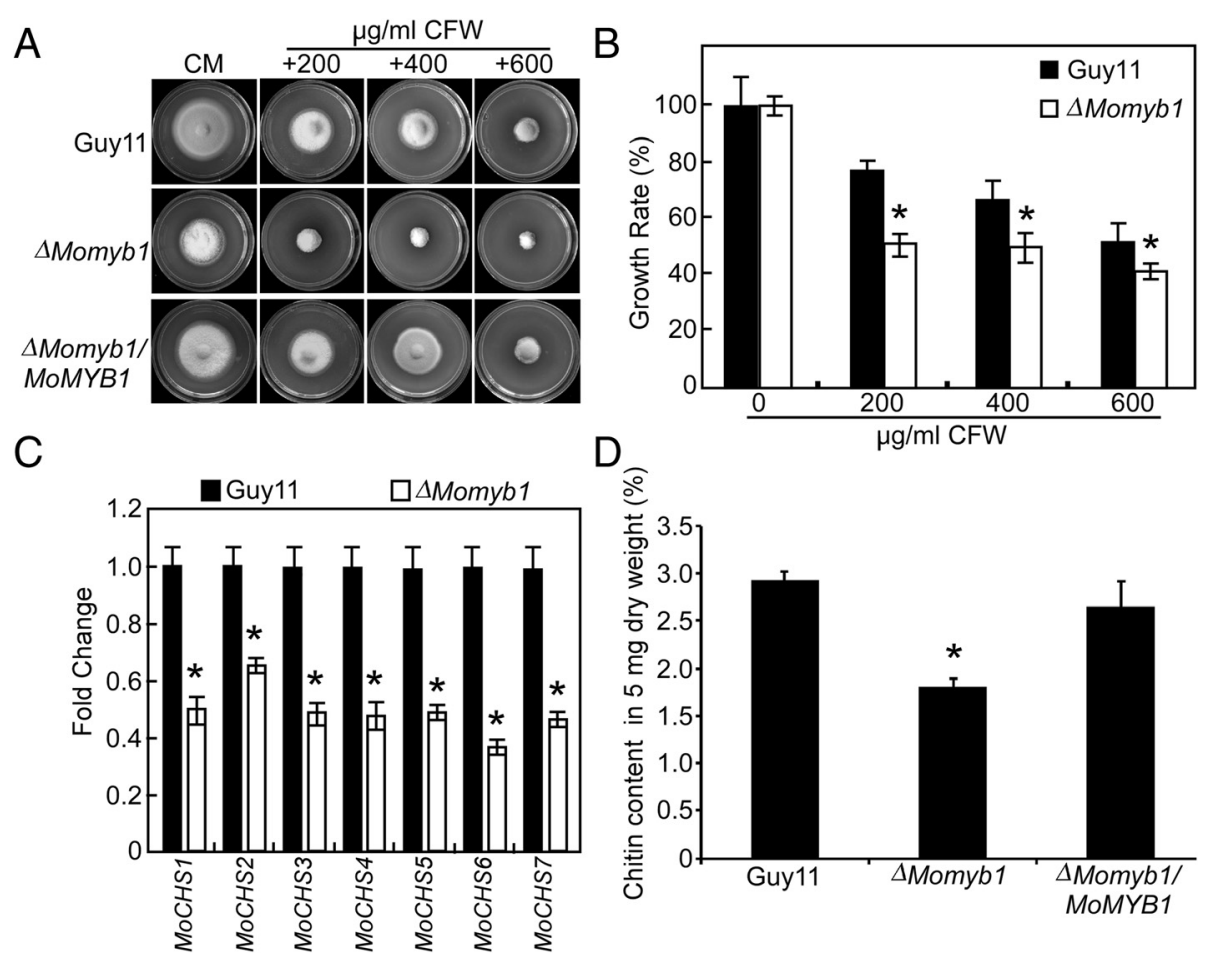

Figure 7 MoMyb1 has a role in cell wall integrity. (A) Wild type Guy11, $\Delta$ Momyb1 mutant and the complemented transformant were incubated on CM plate containing different concentrations of CFW at $28^{\circ} \mathrm{C}$ for 7 days. (B) The growth rate was determined 7 days after incubation at $28^{\circ} \mathrm{C}$ by plotting the percentage of colonies in the presence of various concentrations of CFW against regular CM. (C) The expression levels of seven chitin synthases encoding genes in the $\Delta$ Momyb1 mutant. (D) GlcNa determination shows significantly decreased chitin contents in the $\triangle$ Momyb1 mutant. Data comprise three independent experiments with triple replications each time that yielded similar results. Asterisks were indicated significant differences at $P<0.01$.

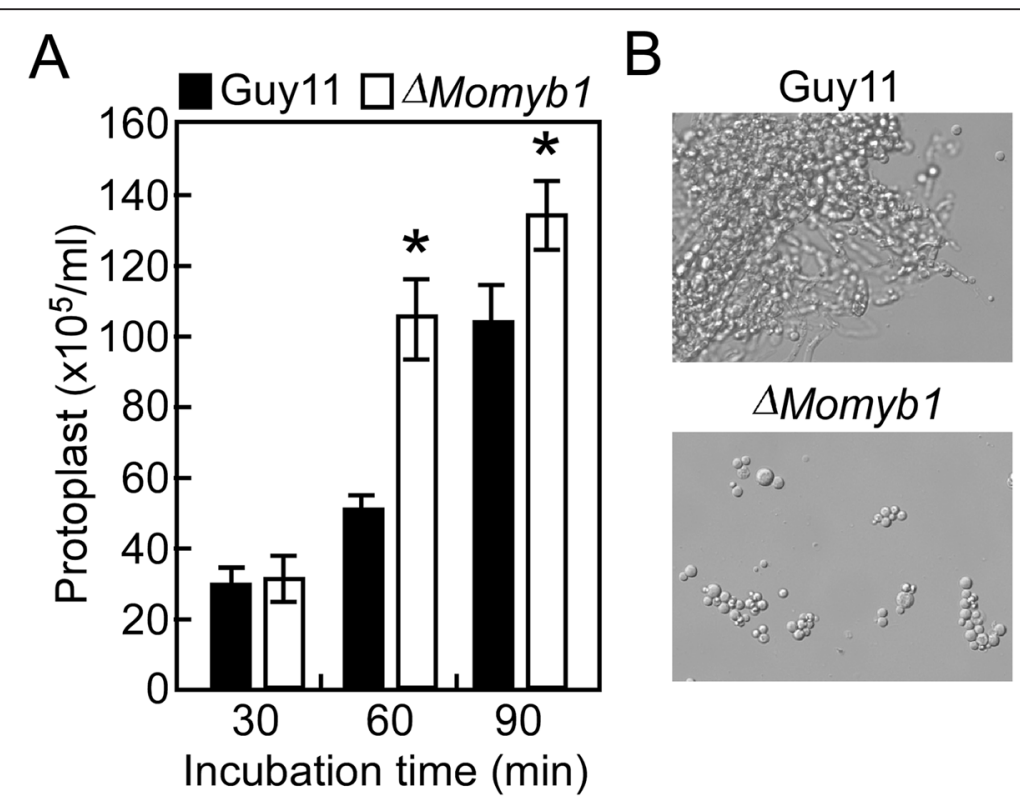

Figure 8 Protoplast release of the wild type Guy11 and $\Delta$ Momyb1 mutant. (A) Protoplast production of Guy 11 and $\Delta M o m y b 1$ mutant treated by cell wall degrading enzyme. Asterisks indicate a significant difference between the mutant and wild-type strain at $P<0.01$. (B) Light microscopic examination of protoplast release after treatment for 60 min and photographed. 
growth, conidiation, stress response, cell wall integrity and virulence.

Conidiogenesis and appressorium development are key steps in the rice blast disease cycle. The fungus has evolved regulatory networks to ensure the correct timing and spatial pattern of these development events [7]. The expression profile of MoMYB1 at hyphal, conidial and infectious stages of $M$. oryzae suggested that MoMyb1 might have a crucial role in conidial development and plant infection. Deletion of MoMYB1 in M. oryzae affected different developmental stages, including hyphal growth, cell wall assembly and penetration. In addition, the $\triangle M o m y b 1$ mutants displayed no conidia and conidiophores, suggesting that deletion of MoMYB1 in M. oryzae completely abolished conidiophore development and thus affected conidium production. Therefore, we conclude that MoMyb1 is a key regulator of M. oryzae for conidium and conidiophore development. qRT-PCR analysis revealed the expression level of five conidiogenesisrelated genes were significantly decreased in the $\Delta M o m y b 1$ mutant, indicating that MoMyb1 probably functions as a key upstream transcription factor in the conidiogenesis signalling pathway to regulate the expression of genes which involved in conidiophore and conidium development. However, whether these conidiogenesis-related genes directly regulated by MoMyb1 need further studies. One surprise result was that the $\Delta$ Momyb1 mutant caused rice blast on rice roots, but not on leaves. Since the infectious mechanism of $M$. oryzae on rice roots has been well clarified [48], we conclude that the pathogenic difference in $\Delta M o m y b 1$ on the two organs is a result of tissue-specific infectious-related development mechanisms.

Cell fate specification is a process of fundamental importance during development. In maize, GL1 encodes a protein containing a Myb DNA-binding domain and is expressed most highly very early during trichome development and is a central regulator of the trichome cell fate decision [49]. Two Myb proteins from fungi, the $C D C 5$ gene product from S. pombe [22] and the FLBD gene product from $A$. nidulans [23] can also control aspects of cell shape, indicating Myb proteins are related to cell wall integrity. Our results show that MoMyb1 is involved in the response to multiple stresses via regulating different signalling pathways including Hog1 pathway, which contributes to osmoregulation. The insensitive or hypersensitive of $\triangle M o m y b 1$ mutant to variety of stresses may also indicate that MoMYB1 is involved in cell wall integrity.

Chitin is an integral part of the fungal cell wall and its synthesis depends on the activity of chitin synthase enzymes. In the present study, the $\Delta M o m y b 1$ deletion mutants showed high sensitivity to the cell wall stressor CFW. In M. oryzae, seven chitin synthases encoding genes were characterized to involve in the development and pathogenicity by affected chitin content [47]. In other phytopathogenic fungi, chitin synthases also play crucial roles in proper regulation of infection-related morphogenesis [50-54]. In the $\Delta$ Momyb1 mutant, the transcriptional levels of seven chitin synthases were down-regulated and the chitin content was also decreased in the mutant, suggesting that MoMyb1 may be a multi-stress regulator of $M$. oryzae that contributes to the loss of pathogenicity. MoMyb1 might directly or indirectly regulate chitin synthesis, thus may disturb the responses of cell wall signalling pathways to different stressors.

\section{Conclusion}

This study demonstrated that MoMyb1 functions as a key regulator that important for vegetative growth, conidiogenesis, stress response and pathogenicity in $M$. ory$z a e$. MoMyb1 is also involved in the maintenance of cell wall integrity that are crucial for the growth and development of the fungus.

\section{Additional files}

\begin{abstract}
Additional file 1: Table S1. Primers used in this study.
Additional file 2: Figure S1. Alignment of gene encoding repeats domain among $M$. oryzae and other organisms. The predicted amino-terminal sequence of MoMyb1 is compared with that of various Myb-like proteins: Aspergillus nidulans FlbD [23]; Homo sapiens c-Myb [41,42]; Arabidopsis thaliana Atr1 [43]; Zea Mays Cl [44]; Schizosaccharomyces pombe Cdc5 [22]; S. cerevisiae Bas1 [45]. The two repeating motifs of $\sim 50$ amino acids are grouped, and the amino acids identical in five or more are showed in grey bars.

Additional file 3: Figure S2. Generation of MOMYB1 deletion mutant. (A) Restriction map of the MOMYB1 genomic region. Disruption constructs containing the homologous sequences flanking the HPH cassette to replace MOMYB1. (B) Southern blot analysis of the wild type (lane 1, 2, 3 and 6) and $\triangle$ Momyb1 mutant (lane 4, 5, 7 and 8) using MOMYB1 and $H P H$ specific probes, respectively. Genomic DNA was digested with EcoRl, Sall and Ndel, respectively. (C) RT-PCR analysis of MOMYB1 in wild type, $\triangle M o m y b 1$ mutants and the complemented transformant. The transcript was lost in the $\Delta$ Momybl mutants.
\end{abstract}

\section{Abbreviations}

qRT-PCR: Quantitative reverse transcription polymerase chain reaction; CFW: Calcofluor white; DNA: Desoxyribonucleic acid; ORF: Open reading frame; BLAST: Basic local alignment search tool; SANT: a putative DNA binding domain in the SWI-SNF and ADA complexes, the transcriptional co-repressor N-CoR and TFIIIB.

\section{Competing interests}

The authors declare that they have no competing interests.

\section{Authors' contributions}

YD, QZ, XL, X Zhang, ZQ, HZ and ZZ carried out the molecular genetic studies, participated in the sequence alignment and drafted the manuscript. YD and X Zhang participated in the sequence alignment. YD, QZ, HZ and ZZ participated in the design of the study and performed the statistical analysis. $\mathrm{HZ}, \mathrm{ZZ}$ and $\mathrm{X}$ Zheng conceived of the study, and participated in its design and coordination. All authors read and approved the final manuscript. 


\section{Acknowledgements}

This research was supported by the National Science Foundation for Distinguished Young Scholars of China (Grant No.31325022 to ZZ), Natural Science Foundation of China (Grant No: 31271998, ZZ, Grant No: 31201471, HZ).

\section{Received: 9 September 2014 Accepted: 4 February 2015 Published online: 19 February 2015}

\section{References}

1. Valent B, Chumley FG. Molecular genetic analysis of the rice blast fungus Magnaporthe grisea. Annu Rev Phytopathol. 1991;29:443-67.

2. Talbot NJ. On the trail of a cereal killer: Exploring the biology of Magnaporthe grisea. Annu Rev Microbiol. 2003;57:177-202.

3. Howard RJ, Ferrari MA, Roach DH, Money NP. Penetration of hard substrates by a fungus employing enormous turgor pressures. Proc Natl Acad Sci USA. 1991;88(24):11281-4.

4. de Jong JC, McCormack BJ, Smirnoff N, Talbot NJ. Glycerol generates turgor in rice blast. Nature. 1997;389(6648):244-5.

5. Pabo CO, Sauer RT. Transcription factors: structural families and principles of DNA recognition. Annu Rev Biochem. 1992;61:1053-95.

6. Li G, Zhou X, Xu JR. Genetic control of infection-related development in Magnaporthe oryzae. Curr Opin Microbiol. 2012;15(6):678-84.

7. Kim S, Park S-Y, Kim KS, Rho H-S, Chi M-H, Choi J, et al. Homeobox transcription factors are required for conidiation and appressorium development in the rice blast fungus Magnaporthe oryzae. PLoS Genet. 2009;5(12):e1000757.

8. Zhang H, Zhao Q, Liu K, Zhang Z, Wang Y, Zheng X. MgCRZ1, a transcription factor of Magnaporthe grisea, controls growth, development and is involved in full virulence. FEMS Microbiol Lett. 2009;293(2):160-9.

9. Guo M, Chen Y, Du Y, Dong Y, Guo W, Zhai S, et al. The bZIP transcription factor MoAP1 mediates the oxidative stress response and is critical for pathogenicity of the rice blast fungus Magnaporthe oryzae. PLoS Pathog. 2011;7(2):e1001302.

10. Qi Z, Wang Q, Dou X, Wang W, Zhao Q, Lv R, et al. MoSwi6, an APSES family transcription factor, interacts with MoMps1 and is required for hyphal and conidial morphogenesis, appressorial function and pathogenicity of Magnaporthe oryzae. Mol Plant Pathol. 2012;13(7):677-89.

11. Zhang H, Zhao Q, Guo X, Guo M, Qi Z, Tang W, et al. Pleiotropic function of the putative zinc-finger protein MoMsn2 in Magnaporthe oryzae. Mol Plant Microbe Interact. 2014:27:446-60

12. Guo M, Guo W, Chen Y, Dong S, Zhang X, Zhang H, et al. The basic leucine zipper transcription factor Moatf1 mediates oxidative stress responses and is necessary for full virulence of the rice blast fungus Magnaporthe oryzae. Mol Plant Microbe Interact. 2010;23(8):1053-68

13. Tang W, Ru Y, Hong L, Zhu Q, Zuo R, Guo X, et al. System-wide characterization of bZIP transcription factor proteins involved in infectionrelated morphogenesis of Magnaporthe oryzae. Environ Microbiol 2014. doi:10.1111/1462-2920.12618

14. Stracke R, Werber M, Weisshaar B. The R2R3-MYB gene family in Arabidopsis thaliana. Curr Opin Plant Biol. 2001;4(5):447-56.

15. Klempnauer KH, Gonda TJ, Bishop JM. Nucleotide sequence of the retroviral leukemia gene $v$-myb and its cellular progenitor c-myb: the architecture of a transduced oncogene. Cell. 1982;31(2 Pt 1):453-63.

16. Jin H, Martin C. Multifunctionality and diversity within the plant MYB-gene family. Plant Mol Biol. 1999;41(5):577-85.

17. Thompson MA, Ramsay RG. Myb: an old oncoprotein with new roles. Bioessays. 1995;17(4):341-50.

18. Rosinski JA, Atchley WR. Molecular evolution of the Myb family of transcription factors: evidence for polyphyletic origin. J Mol Evol. 1998:46(1):74-83.

19. Weston K. Myb proteins in life, death and differentiation. Curr Opin Genet Dev. 1998:8(1):76-81

20. Ito M. Conservation and diversification of three-repeat Myb transcription factors in plants. J Plant Res. 2005;118(1):61-9.

21. Martin C, PazAres J. MYB transcription factors in plants. Trends Genet. 1997;13(2):67-73.

22. Ohi R, McCollum D, Hirani B, Den Haese GJ, Zhang X, Burke JD, et al. The Schizosaccharomyces pombe cdc5+ gene encodes an essential protein with homology to c-Myb. EMBO J. 1994;13(2):471-83.

23. Wieser J, Adams TH. flbD encodes a Myb-like DNA-binding protein that coordinates initiation of Aspergillus nidulans conidiophore development. Genes Dev. 1995;9(4):491-502.
24. Ohi R, Feoktistova A, McCann S, Valentine V, Look AT, Lipsick JS, et al. Myb-related Schizosaccharomyces pombe cdc5p is structurally and functionally conserved in eukaryotes. Mol Cell Biol. 1998;18(7):4097-108.

25. Talbot NJ, Ebbole DJ, Hamer JE. Identification and characterization of MPGI, a gene involved in pathogenicity from the rice blast fungus Magnaporthe grisea. Plant Cell. 1993;5:1575-90.

26. Zhang H, Liu K, Zhang X, Song W, Zhao Q, Dong Y, et al. A two-component histidine kinase, MOSLN1, is required for cell wall integrity and pathogenicity of the rice blast fungus. Magnaporthe oryzae. Curr Genet. 2010;56(6):517-28.

27. Zhang H, Liu K, Zhang X, Tang W, Wang J, Guo M, et al. Two phosphodiesterase genes, PDEL and PDEH, regulate development and pathogenicity by modulating intracellular cyclic AMP levels in Magnaporthe oryzae. PLoS One. 2011;6(2):e17241.

28. Bruno KS, Tenjo F, Li L, Hamer JE, Xu J-R. Cellular localization and role of kinase activity of PMK1 in Magnaporthe grisea. Eukaryot Cell. 2004;3(6):1525-32.

29. Dufresne M, Osbourn AE. Definition of tissue-specific and general requirements for plant infection in a phytopathogenic fungus. Mol Plant-Microbe Interact. 2001;14(3):300-7

30. Chen Y, Zhai S, Zhang H, Zuo R, Wang J, Guo M, et al. Shared and distinct functions of two Gti1/Pac2 family proteins in growth, morphogenesis and pathogenicity of Magnaporthe oryzae. Environ Microbiol. 2014;16(3):788-801.

31. Bulik DA, Olczak M, Lucero HA, Osmond BC, Robbins PW, Specht CA. Chitin synthesis in Saccharomyces cerevisiae in response to supplementation of growth medium with glucosamine and cell wall stress. Eukaryot Cell. 2003;2(5):886-900

32. Song W, Dou X, Qi Z, Wang Q, Zhang X, Zhang H, et al. R-SNARE homolog MoSec22 is required for conidiogenesis, cell wall integrity, and pathogenesis of Magnaporthe oryzae. PloS One. 2010;5(10):e13193.

33. Jeon J, Goh J, Yoo S, Chi M-H, Choi J, Rho H-S, et al. A putative MAP kinase kinase kinase, $M C K 1$, is required for cell wall integrity and pathogenicity of the rice blast fungus. Magnaporthe oryzae. Mol Plant-Microbe Interact. 2008;21(5):525-34

34. Dou X, Wang Q, Qi Z, Song W, Wang W, Guo M, et al. MoVam7, a conserved SNARE involved in vacuole assembly, is required for growth, endocytosis, ROS accumulation, and pathogenesis of Magnaporthe oryzae. Plos One. 2011;6(1):e16439.

35. Sambrook J, Fritsch EF, Maniatis T. Molecular Cloning: a Laboratory Manual. Cold Spring Harbor, NY: Cold Spring Harbor Laboratory Press; 1989.

36. Livak KJ, Schmittgen TD. Analysis of relative gene expression data using real-time quantitative PCR and the 2(T)(-Delta Delta C) method. Methods. 2001;25(4):402-8

37. Dean RA, Talbot NJ, Ebbole DJ, Farman ML, Mitchell TK, Orbach MJ, et al. The genome sequence of the rice blast fungus Magnaporthe grisea. Nature. 2005:434:989-6.

38. McGinnis S, Madden TL. BLAST: at the core of a powerful and diverse set of sequence analysis tools. Nucleic Acids Res. 2004;32(Web Server issue):W20-5.

39. Thompson JD, Higgins DG, Gibson TJ. CLUSTAL W: improving the sensitivity of progressive multiple sequence alignment through sequence weighting, position-specific gap penalties and weight matrix choice. Nucleic Acids Res. 1994:22(22):4673-80.

40. Aasland R, Stewart AF, Gibson T. The SANT domain: A putative DNA-binding domain in the SWI-SNF and ADA complexes, the transcriptional corepressor N-CoR and TFIIIB. Trends Biochem Sci. 1996;21(3):87-8.

41. Majello B, Kenyon LC, Dalla-Favera R. Human c-myb protooncogene: nucleotide sequence of cDNA and organization of the genomic locus. Proc Natl Acad Sci USA. 1986;83(24):9636-40.

42. Slamon DJ, Boone TC, Murdock DC, Keith DE, Press MF, Larson RA, et al. Studies of the human c-myb gene and its product in human acute leukemias. Science. 1986:233(4761):347-51.

43. Bender J, Fink GR. A Myb homologue, ATR1, activates tryptophan gene expression in Arabidopsis. Proc Natl Acad Sci USA. 1998;95(10):5655-60.

44. Paz-Ares J, Ghosal D, Wienand U, Peterson PA, Saedler $H$. The regulatory c1 locus of Zea mays encodes a protein with homology to myb proto-oncogene products and with structural similarities to transcriptional activators. EMBO J. 1987;6(12):3553-8.

45. Tice-Baldwin K, Fink GR, Arndt KT. BAS1 has a Myb motif and activates HIS4 transcription only in combination with BAS2. Science. 1989;246(4932):931-5.

46. Zhou Z, Li G, Lin C, He C. Conidiophore stalk-less1 encodes a putative zincfinger protein involved in the early stage of conidiation and mycelial infection in Magnaporthe oryzae. Mol Plant-Microbe Interact. 2009;22(4):402-10. 
47. Kong L, Yang J, Li G, Qi L, Zhang Y, Wang C, et al. Different chitin synthase genes are required for various developmental and plant infection processes in the rice blast fungus Magnaporthe oryzae. PLoS Pathog. 2012;8(2):e1002526.

48. Tucker SL, Besi MI, Galhano R, Franceschetti M, Goetz S, Lenhert S, et al. Common genetic pathways regulate organ-specific infection-related development in the rice blast fungus. Plant Cell. 2010;22(3):953-72.

49. Larkin JC, Oppenheimer DG, Lloyd AM, Paparozzi ET, Marks MD. Roles of the glabrous1 and transparent testa glabra genes in Arabidopsis trichome Development. Plant Cell. 1994;6(8):1065-76.

50. Soulie MC, Piffeteau A, Choquer M, Boccara M, Vidal-Cros A. Disruption of Botrytis cinerea class I chitin synthase gene Bcchs 1 results in cell wall weakening and reduced virulence. Fungal Genet Biol. 2003;40(1):38-46.

51. Soulie MC, Perino C, Piffeteau A, Choquer M, Malfatti P, Cimerman A, et al. Botrytis cinerea virulence is drastically reduced after disruption of chitin synthase class III gene (Bcchs3a). Cell Microbiol. 2006;8(8):1310-21.

52. Madrid MP, Di Pietro A, Roncero MIG. Class V chitin synthase determines pathogenesis in the vascular wilt fungus Fusarium oxysporum and mediates resistance to plant defence compounds. Mol Microbiol. 2003;47(1):257-66.

53. Martin-Udiroz M, Madrid MP, Roncero MIG. Role of chitin synthase genes in Fusarium oxysporum. Microbiol-Sgm. 2004;150:3175-87.

54. Odenbach D, Thines E, Anke H, Foster AJ. The Magnaporthe grisea class VII chitin synthase is required for normal appressorial development and function. Mol Plant Pathol. 2009:10(1):81-94.

\section{Submit your next manuscript to BioMed Central and take full advantage of:}

- Convenient online submission

- Thorough peer review

- No space constraints or color figure charges

- Immediate publication on acceptance

- Inclusion in PubMed, CAS, Scopus and Google Scholar

- Research which is freely available for redistribution 